\title{
Resolution of trigeminal neuralgia by coil embolization of a persistent primitive trigeminal artery aneurysm
}

\author{
Travis R Ladner, Moneeb Ehtesham, Brandon J Davis, Imad S Khan, Mayshan Ghiassi, \\ Mahan Ghiassi, Robert J Singer
}

Department of Neurosurgery, Vanderbilt University, Nashville, Tennessee, USA

\section{Correspondence to}

Travis R Ladner,

travis.r.ladner@vanderbilt.edu

\section{SUMMARY}

The persistent primitive trigeminal artery (PTA) is a rare anastomosis between the carotid artery and basilar artery. While most PTAs are asymptomatic, lateral variants can occasionally compress the trigeminal nerve and precipitate trigeminal neuralgia. Aneurysms of the PTA are exceptionally rare in the literature and have not previously been associated with trigeminal neuralgia. We present the first case of an aneurysm of the PTA causing trigeminal neuralgia. The patient underwent coil embolization of the aneurysm which relieved her symptoms. We propose embolization as a viable therapeutic option for the resolution of trigeminal neuralgia when the condition is secondary to irritation by the high velocity pulsatile flow of an aneurysm.

\section{BACKGROUND}

Trigeminal neuralgia is most typically caused by vascular compression of the trigeminal nerve by nearby cerebral arteries, especially the superior cerebral artery, posterior communicating artery and persistent primitive trigeminal artery (PTA). Trigeminal neuralgia can also be caused by vascular compression of the trigeminal nerve by aneurysms of nearby vessels. ${ }^{1-5}$ Curiously, despite the close anatomical proximity between the PTA and the trigeminal nerve, there are no reported cases of trigeminal neuralgia secondary to PTA aneurysms. To date, there are only around 40 previous reports of such an aneurysm in the world literature, with the most common presenting symptoms being abducens palsy and headache. ${ }^{6}$ We report the first account of the resolution of trigeminal neuralgia following coil embolization of a PTA aneurysm.

\section{CASE PRESENTATION}

A 66-year-old woman with a history of diabetes mellitus type 2 was referred to our neurovascular clinic for consultation regarding sudden-onset headache and left facial pain with a V1/V2 distribution. There was no associated loss of consciousness, no altered mental status, no nuchal rigidity and no focal neurological deficits. She endorsed a family history of ruptured brain aneurysms.

\section{INVESTIGATIONS}

CT performed in an outside emergency department revealed an unruptured calcified saccular aneurysm, without evidence of subarachnoid hemorrhage, originating off the left internal carotid artery (ICA).
Given this imaging and clinical history, the suspicion for subarachnoid hemorrhage was low. The patient therefore underwent diagnostic catheter angiography which revealed a saccular aneurysm arising from the left PTA, measuring $7.0 \times 5.0 \mathrm{~mm}$, with a relatively broad-based $2.8 \mathrm{~mm}$ neck. The diameter of the PTA segment proximal to the aneurysm was $2.3 \mathrm{~mm}$ (figure 1).

\section{TREATMENT}

After viewing these images, a 0.010 inch microcatheter was transnavigated over a 0.014 inch wire to select the PTA aneurysm. A series of coils was placed in the aneurysm, achieving total occlusion (figure 2).

\section{OUTCOME AND FOLLOW-UP}

The patient's facial pain was immediately relieved following surgery. She was monitored for $24 \mathrm{~h}$ on heparin and discharged to home on clopidogrel and aspirin. At follow-up 6 months after surgery the patient reported complete resolution of her

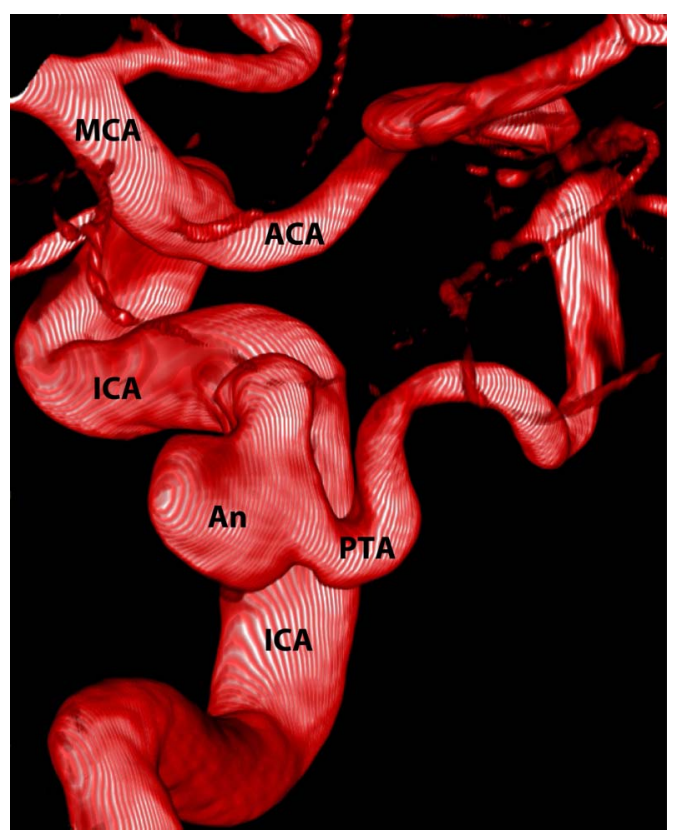

Figure 1 Three-dimensional digital subtraction angiography demonstrating PTA aneurysm before embolization. ACA, anterior cerebral artery; $A n$, aneurysm; ICA, internal carotid artery; MCA, middle cerebral artery; PTA, persistent primitive trigeminal artery. 


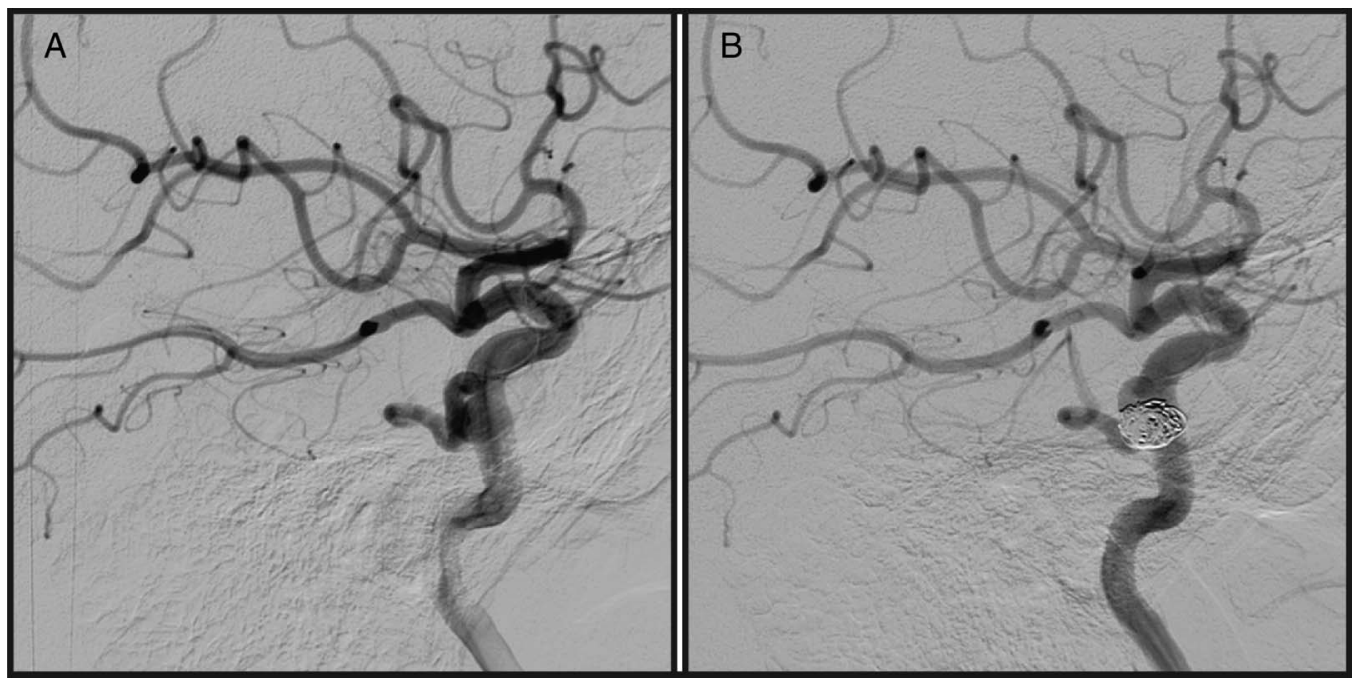

Figure 2 Digital subtraction angiography demonstrating persistent primitive trigeminal artery aneurysm (A) before and (B) after embolization in lateral view.

symptoms, with no facial pain or dysesthesia. Magnetic resonance angiography demonstrated sustained embolization of the aneurysm with no recanalization (figure 3 ).

\section{DISCUSSION}

The PTA is a very rare anastomosis between the ICA and basilar artery (BA), seen in between $0.1 \%$ and $1 \%$ of all cerebral angiograms. $^{7}$ It is the most common of the persistent fetal carotidvertebrobasilar anastomoses (figure 4). The PTA originates from the precavernous ICA, just proximal to the meningohypophyseal trunk, and can then course either medially or laterally between the ICA and BA. Both variants are equally common. ${ }^{8}$ The medial variant courses posteromedially through the sella tursica in its own groove, pierces the clival dura at the dorsum sellae and anastomoses with the BA. ${ }^{7} 9$

The lateral variant exits through the cavernous sinus and courses between the trigeminal nerve and abducens nerve in a groove of the posterior petrosal process, coursing toward
Meckel's cave and subsequently anastomosing with the BA. ${ }^{7}$ Because of its anatomical location, the lateral PTA variant is therefore more likely to compress the trigeminal nerve and cause symptoms. ${ }^{9}$ Our patient's PTA followed a lateral course, which supports the hypothesis that trigeminal neuralgia was secondary to an aneurysm of this vessel (figure 3).

PTAs are also characterized by the origination of the posterior cerebral artery. ${ }^{8}$ A Saltzman type 1 has an absent ipsilateral posterior communicating artery while a Saltzman type 2 has a fetal origin ipsilateral posterior cerebral artery directly off the ICA. Our patient's PTA would therefore be classified as a lateral variant Saltzman type 2.

Aneurysms arise from the PTA relatively frequently, possibly due to turbulent flow associated with the direct branching of the PTA off the ICA. Approximately 15\% of cases of PTA have an associated aneurysm, with the most common location being the bifurcation of the cavernous segment of the ICA and the PTA. ${ }^{6}$ Abducens palsy is the most common neurological deficit,
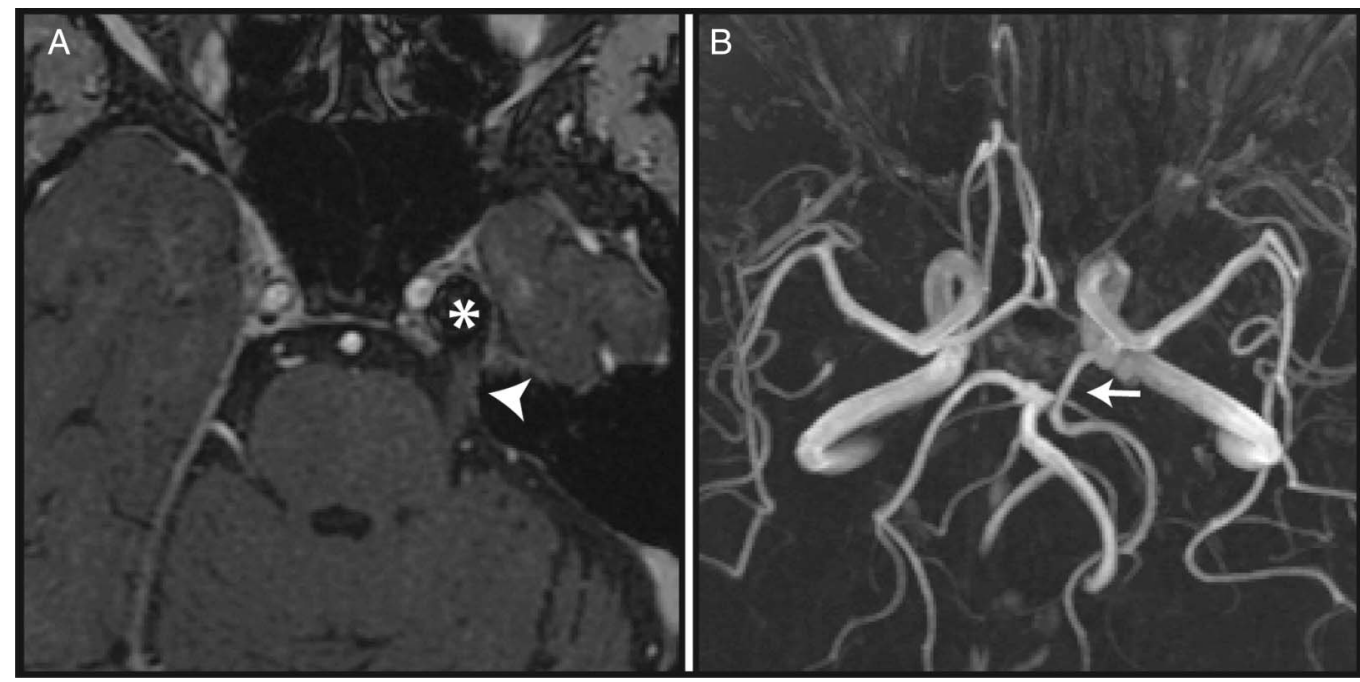

Figure 3 Follow-up axial magnetic resonance angiography demonstrating the relationship of the vasculature to other cerebral structures. (A) Signal drop-out near the left internal carotid artery indicates artefact from coils, giving the position of the aneurysm (asterisk) relative to the trigeminal nerve (arrowhead). (B) The course of the persistent primitive trigeminal artery (arrow) in relation to cerebral structures indicates lateral Saltzman type 2 variant. 


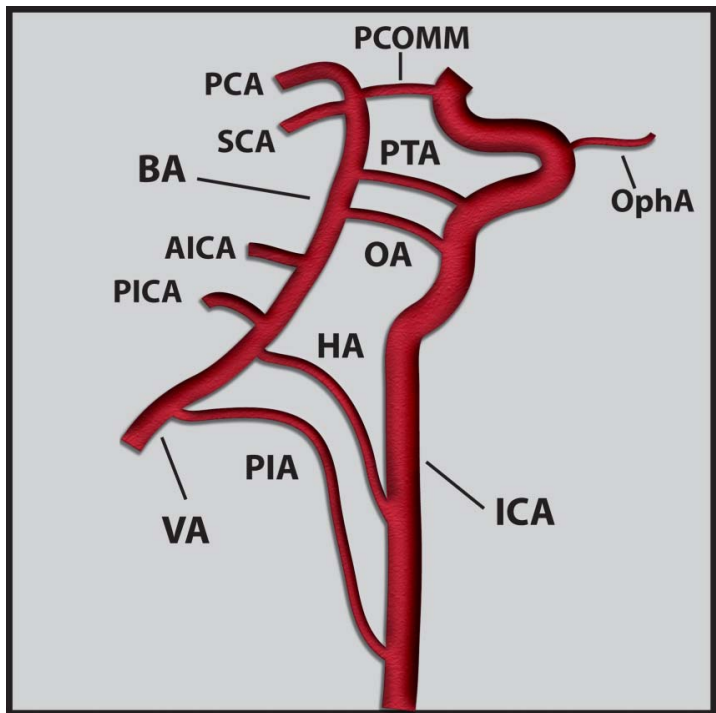

Figure 4 Persistent fetal carotid-vertebrobasilar anastomoses. AICA, anterior inferior cerebellar artery; BA, basilar artery; $H A$, hypoglossal artery; ICA, internal carotid artery; $O A$, otic artery; OphA, ophthalmic artery; PCA, posterior cerebral artery; PCOMM, posterior communicating artery; PIA, proatlantal intersegmental artery; PICA, posterior inferior cerebellar artery; PTA, persistent primitive trigeminal artery; SCA, superior cerebellar artery; VA, vertebral artery.

which is explained by the proximity of ICA-PTA bifurcation aneurysms to the abducens nerve.

Curiously, although the PTA courses alongside the trigeminal nerve in lateral variants, trigeminal neuralgia has not previously been reported in the setting of a PTA aneurysm. Previously reported PTA aneurysms most likely were not of an appropriate size, orientation and/or location to involve the trigeminal nerve and possibly were not pulsatile enough to irritate it. Our patient developed trigeminal neuralgia because the aneurysm projected posterolaterally, compressing the V1 and V2 segments of the trigeminal nerve near the cavernous sinus. The abducens nerve was spared and therefore the patient did not exhibit abducens palsy. This aneurysm was calcified, suggesting it had been in this location for a while. Therefore, her symptoms are less likely explained solely by mass effect but rather a spontaneous change in the hemodynamic status of the aneurysm causing high velocity pulsatile flow.

Trigeminal neuralgia has been associated with aneurysms of nearby vessels, most commonly arising from the posterior communicating artery. Most of these aneurysms have been treated with surgical clipping. Coiling does not immediately decrease mass effect like surgical clipping. Rather, coiling eliminates high velocity pulsatile flow through the aneurysm across the surface of the compressed nerve and can quickly relieve symptoms. This has been shown in aneurysm-induced oculomotor palsy. In a series of 21 patients with oculomotor palsy undergoing coil embolization of a posterior communicating artery aneurysm, $91 \%$ had resolution of symptoms. ${ }^{10}$

One case of coil embolization of a small fusiform P2 aneurysm causing trigeminal neuralgia was found in the literature. ${ }^{1}$
The patient's symptoms diminished over the course of 1 week after embolization. Our case provides additional support that aneurysm-induced trigeminal neuralgia is amenable to treatment by endovascular methods. Had we been unsuccessful, surgical clipping would have been considered as a second option.

To our knowledge, this is the first report of trigeminal neuralgia in the setting of a PTA aneurysm. We propose high velocity pulsatile flow through the aneurysm across the surface of the trigeminal ganglion as the etiology of her pain. Coil embolization was effective in securing the aneurysm and alleviating the symptoms.

\section{Learning points}

- A persistent primitive trigeminal artery should be considered in a patient presenting with trigeminal neuralgia.

- Trigeminal neuralgia can also be caused by vascular compression of the trigeminal nerve by aneurysms of nearby vessels.

- Embolization may be an effective treatment for trigeminal neuralgia secondary to a nearby aneurysm.

Contributors TRL wrote the original manuscript. ME operated on the patient and helped write the manuscript. BJD obtained diagnostic images of the patient and helped revise the manuscript. ISK obtained informed consent from the patient and revised the manuscript. MayG and MahG helped revise the manuscript. RJS operated on the patient, provided images and revised the manuscript.

\section{Competing interests None.}

Patient consent Obtained.

Provenance and peer review Not commissioned; externally peer reviewed.

\section{REFERENCES}

1 Roh HG, Kim SS, Han H, et al. Endovascular treatment of posterior cerebral artery aneurysms using detachable coils. Neuroradiology 2008;50:237-42.

2 Terao S, Hara K, Yoshida $\mathrm{K}$, et al. A giant internal carotid-posterior communicating artery aneurysm presenting with atypical trigeminal neuralgia and facial nerve palsy in a patient with autosomal dominant polycystic kidney disease: a case report. Surg Neurol 2001;56:127-31.

3 Ildan F, Göçer Al, Bağdatoğlu $\mathrm{H}$, et al. Isolated trigeminal neuralgia secondary to distal anterior inferior cerebellar artery aneurysm. Neurosurg Rev 1996;19:43-6.

4 Leopold NA, Hirsh LF, Ray T. Paroxysmal facial neuralgia secondary to a posterior communicating artery aneurysm. Surg Neurol 1980;14:221-3.

5 Sobel D, Norman D, Yorke CH, et al. Radiography of trigeminal neuralgia and hemifacial spasm. AJR Am J Roentgenol 1980;135:93-5.

6 Kai Y, Ohmori Y, Watanabe $M$, et al. Coil embolization of an aneurysm located at the trunk of the persistent primitive trigeminal artery. Neurol Med Chir (Tokyo) 2011;51:361-4

7 Luh GY, Dean BL, Tomsick TA, et al. The persistent fetal carotid-vertebrobasilar anastomoses. AJR Am J Roentgenol 1999;172:1427-32.

8 Uchino A, Sawada A, Takase Y, et al. MR Angiography of anomalous branches of the internal carotid artery. AJR 2003;181:1409-14.

9 de Bondt B-J, Stokroos R, Casselman J. Persistent trigeminal artery associated with trigeminal neuralgia: hypothesis of neurovascular compression. Neuroradiology 2007;49:23-6.

10 Hanse MCJ, Gerrits MCF, Van Rooij WJ, et al. Recovery of posterior communicating artery aneurysm-induced oculomotor palsy after coiling. AJNR Am J Neuroradiol 2008:29:988-90. 


\section{Clinical neurology}

Copyright 2013 BMJ Publishing Group. All rights reserved. For permission to reuse any of this content visit http://group.bmj.com/group/rights-licensing/permissions.

BMJ Case Report Fellows may re-use this article for personal use and teaching without any further permission.

Become a Fellow of BMJ Case Reports today and you can:

- Submit as many cases as you like

- Enjoy fast sympathetic peer review and rapid publication of accepted articles

- Access all the published articles

- Re-use any of the published material for personal use and teaching without further permission

For information on Institutional Fellowships contact consortiasales@bmjgroup.com

Visit casereports.bmj.com for more articles like this and to become a Fellow 